\title{
3D Visibility Representations by Regular Polygons
}

\author{
Jan Štola \\ Department of Applied Mathematics, Charles University \\ Malostranské nám. 25, Prague, Czech Republic \\ Jan.Stola@mff .cuni.cz
}

\begin{abstract}
We study 3D visibility representations of complete graphs where vertices are represented by equal regular polygons lying in planes parallel to the $x y$-plane. Edges correspond to the $z$-parallel visibility among these polygons.

We improve the upper bound on the maximum size of a complete graph with a $3 \mathrm{D}$ visibility representation by regular $n$-gons from $2^{O(n)}$ to $O\left(n^{4}\right)$.
\end{abstract}

\section{Introduction}

In this paper we study 3D visibility drawings that represent vertices by twodimensional sets placed in planes parallel to the $x y$-plane. Two vertices are connected by an edge if and only if they can see each other in the direction that is orthogonal to their planes, i.e., parallel to the $z$-axis.

This type of representation was introduced as a generalization of the $2 \mathrm{D}$ visibility drawing. The $2 \mathrm{D}$ rectangle visibility drawing received a wide attention because of its connection to VLSI routing and circuit board layout 78 .

The representation of vertices by rectangles remains popular also in the 3D visibility drawing. A lot of papers are focused on the maximum size of a complete graph with a $3 \mathrm{D}$ visibility representation by rectangles. Rote and Zelle provide a representation of $K_{22}$ (see [6]). On the other hand, Bose et al. 4 showed that no complete graph with more than 102 vertices has such a representation. This result was then improved to 55 by Fekete et al. 3] and recently by Stola [5] to 50 .

If the vertices are represented by unit squares then the largest complete graph with this type of representation is $K_{7}$ according to [3]. This is the only exact result known about representations by equal regular $n$-gons. Only estimates are known for $n \neq 4$. Babilon et al. 2 show that $K_{14}$ can be represented by equal triangles. They also present a lower bound $\left\lfloor\frac{n+1}{2}\right\rfloor+2$ on the maximum size of a complete graph with a $3 \mathrm{D}$ visibility representation by equal regular $n$-gons. Stola [1] then moved this bound to $n+1$. The first upper bound $2^{2^{n}}$ was given by Babilon et al. 2]. This doubly-exponential estimate was improved by Stola [1] to an exponential $\left(\begin{array}{c}6 n-3 \\ 3 n-1\end{array}\right)-3 \approx 2^{6 n}$. The main result of this paper is another significant improvement of this bound. We present a polynomial upper bound $O\left(n^{4}\right)$. 


\section{Preliminaries}

Let $P$ be a regular $n$-gon inscribed in a unit circle (with the center $c$ ). Let $v_{0}, v_{1}, \ldots, v_{n}=v_{0}$ be the vertices of $P, s_{0}=\overline{v_{0} v_{1}}, \ldots, s_{n-1}=\overline{v_{n-1} v_{n}}, s_{n}=s_{0}$ the sides of $P, m_{i}$ the center of $s_{i}$ and $p_{i}$ the half-line $\overrightarrow{c m_{i}}$. If $P_{i}$ is a copy of $P$ (shifted by a vector $\boldsymbol{w}_{\boldsymbol{i}}$ ) then we denote its vertices by $v_{j}^{i}$ and the sides by $s_{j}^{i}$.

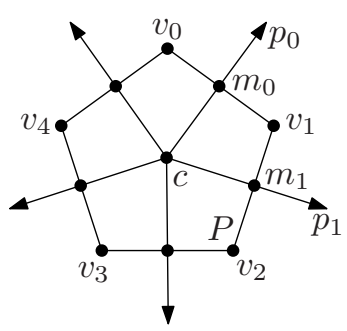

(a)

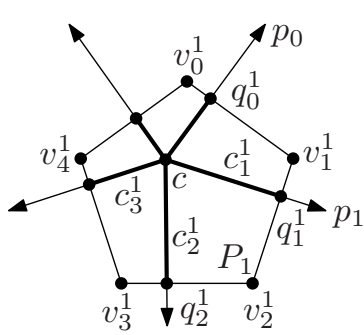

(b)

Fig. 1.

The distance of $v_{j}$ and $p_{j}$ is $\sin (\pi / n)$, similarly $\operatorname{dist}\left(v_{j}, p_{j-1}\right)=\sin (\pi / n)$ and $\operatorname{dist}\left(s_{j}, c\right)=\cos (\pi / n)$. Hence, if $\left|\boldsymbol{w}_{\boldsymbol{i}}\right|<\sin (\pi / n)$ then $v_{j}^{i}$ (the shifted copy of $v_{j}$ ) remains in the angle $\widehat{m_{j-1} c m_{j}}$. If in addition $\left|\boldsymbol{w}_{\boldsymbol{i}}\right|<\cos (\pi / n)$ then $s_{j}^{i}$ intersects $p_{j}$.

Definition 1. Let $\left\{P_{i}, P_{i}=P+\boldsymbol{w}_{\boldsymbol{i}}\right\}$ be the set of shifted copies of a regular $n$-gon $P$ (inscribed in a unit circle). We say that this set is a short-distance set if $\forall i:\left|\boldsymbol{w}_{\boldsymbol{i}}\right|<\min (\sin (\pi / n), \cos (\pi / n))$.

The definition of a short-distance set requires a reference polygon $P$ that is close to every polygon from the set. If the polygons $P_{i}=P+\boldsymbol{w}_{\boldsymbol{i}}$ are far from $P$ but close to each other, i.e., $\forall i, j:\left|\boldsymbol{w}_{\boldsymbol{i}}-\boldsymbol{w}_{\boldsymbol{j}}\right|<\min (\sin (\pi / n), \cos (\pi / n))$ then they also form a short-distance set because we can take any $P_{i}$ as a reference polygon in this case.

For a polygon $P_{i}$ from a short-distance set we can define $q_{j}^{i}=p_{j} \cap s_{j}^{i}$ and $c_{j}^{i}=\operatorname{dist}\left(c, q_{j}^{i}\right)$ (see Figure 10). We call the $n$-tuple $\left(c_{j}^{i}\right)_{j=1}^{n}$ the coordinates of $P_{i}$.

Every polygon can be reconstructed from its coordinates (see Figure 2). If $H_{j}^{i}$ is the half-plane with its boundary line $h_{j}^{i}$ such that $c \in H_{j}^{i}, h_{j}^{i} \perp p_{j}$ and $\operatorname{dist}\left(h_{j}^{i}, c\right)=c_{j}^{i}$ then $P_{i}=\bigcap_{j=1}^{n} H_{j}^{i}$. Therefore the intersection $P_{i} \cap P_{k}=$ $\bigcap_{j=1}^{n}\left(H_{j}^{i} \cap H_{j}^{k}\right)$ can be described by coordinates $\left(\min \left(c_{j}^{i}, c_{j}^{k}\right)\right)_{j=1}^{n}$.

We assume in the sequel that $P$ is a regular $n$-gon inscribed in a unit circle and $\left\{P_{i}=P+\boldsymbol{w}_{\boldsymbol{i}}, i=1, \ldots, m\right\}$ is a $3 \mathrm{D}$ visibility representation of a complete graph $K_{m}$. We assume that the $z$-coordinate of $P_{i}$ is $i$ but we use it to identify polygons that can block visibility between other polygons only. Otherwise, we ignore the $z$-coordinate and work with the polygons as if they were in the same 


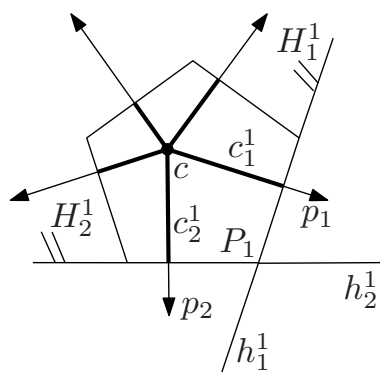

Fig. 2. Reconstruction of the polygon $P_{1}$ from its coordinates

$x y$-parallel plane. Formally, these operations represent operations over orthogonal projections of the relevant objects (points, lines, polygons) into a common $x y$-parallel plane and the projection of the results (for example, intersection points) into individual planes of the polygons.

Lemma 1. Polygons $P_{i}$ and $P_{k}$ can see each other if and only if there exists $l$ such that $\forall j, i<j<k:\left(c_{l}^{j}<\min \left(c_{l}^{i}, c_{l}^{k}\right)\right.$ or $\left.c_{l+1}^{j}<\min \left(c_{l+1}^{i}, c_{l+1}^{k}\right)\right)$.

Proof. $Q=P_{i} \cap P_{k}$ is a polygon given by coordinates $\left(\min \left(c_{j}^{i}, c_{j}^{k}\right)\right)_{j=1}^{n}$. Let $Q_{l}$ be the intersection of $Q$ with the angle $\widehat{m_{l} c m_{l+1}}$ and $q_{l}$ be the (only) vertex of $Q$ in $\widehat{m_{l} c m_{l+1}}$.

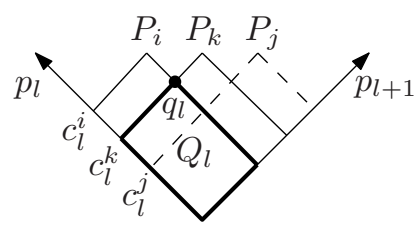

(a)

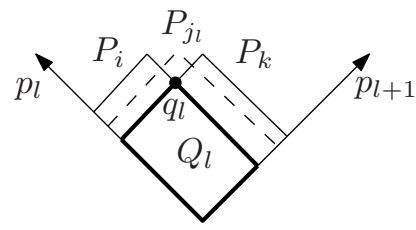

(b)

Fig. 3.

If $c_{l}^{j}<\min \left(c_{l}^{i}, c_{l}^{k}\right)$ or $c_{l+1}^{j}<\min \left(c_{l+1}^{i}, c_{l+1}^{k}\right)$ then $P_{j}$ doesn't block the visibility of $P_{i}$ and $P_{k}$ in the neighborhood of $q_{l}$, see Figure 3 a. Hence, if for a fixed $l$ this condition holds for all polygons $P_{j}$ between $P_{i}$ and $P_{k}$ then $P_{i}$ and $P_{k}$ can see each other in the neighborhood of $q_{l}$.

On the other hand, if $\forall l \exists j_{l}: i<j_{l}<k, c_{l}^{j_{l}} \geq \min \left(c_{l}^{i}, c_{l}^{k}\right)$ and $c_{l+1}^{j_{l}} \geq$ $\min \left(c_{l+1}^{i}, c_{l+1}^{k}\right)$ then $P_{j_{l}}$ blocks the visibility of $P_{i}$ and $P_{k}$ in the angle $\widehat{m_{l} c m_{l+1}}$, see Figure 3b. Therefore $P_{i}$ cannot see $P_{k}$.

Lemma 1 describes a sufficient and necessary condition for the visibility between two polygons from a short-distance set. If we shift the polygon $P_{i}$ by a sufficiently small vector then we don't break any of the strict inequalities in Lemma 1. In 
other words, the shifted polygon can see all polygons that the original polygon can see. Therefore we can replace the original polygon $P_{i}$ by the shifted one without breaking the completeness of the represented graph. This observation allows us to assume in the sequel that $j$-th coordinates of polygons are distinct, i.e., $\forall i, j, k, i \neq k: c_{j}^{i} \neq c_{j}^{k}$.

Lemma 2. Let $P_{i}$ be a regular $n$-gon with coordinates $\left(c_{j}^{i}\right)_{j=1}^{n}$ and $P_{k}=P_{i}+\boldsymbol{w}$ a shifted copy of $P_{i}$ with coordinates $\left(c_{j}^{k}\right)_{j=1}^{n}$. If $n$ is even then there are exactly $n / 2$ adjacent coordinates with $\operatorname{sgn}\left(c_{j}^{k}-c_{j}^{i}\right)=1$ and $n / 2$ adjacent coordinates with the opposite signum. If $n$ is odd then there are $\lfloor n / 2\rfloor$ or $\lceil n / 2\rceil$ adjacent coordinates with $\operatorname{sgn}\left(c_{j}^{k}-c_{j}^{i}\right)=1$ and the rest with the opposite signum.

Proof. The length of the orthogonal projection of $\boldsymbol{w}$ into a line containing $p_{j}$ is $\left|c_{j}^{k}-c_{j}^{i}\right|$. The difference $c_{j}^{k}-c_{j}^{i}$ is positive (resp. negative) if this projection of $\boldsymbol{w}$ has the same (resp. the opposite) orientation as $p_{j}$.

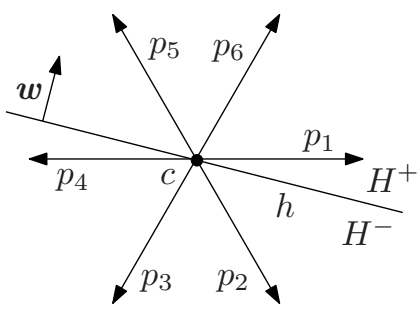

(a)

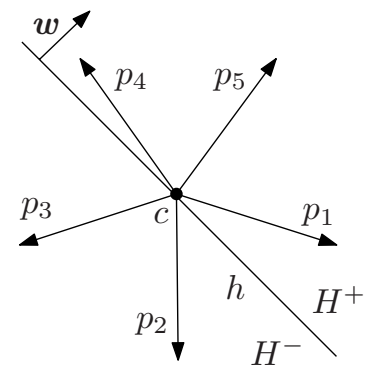

(b)

Fig. 4.

Let $h$ be a line such that $h \perp \boldsymbol{w}$ and $c \in h$. $h$ divides the plane into half-planes $H^{+}$and $H^{-}$. Let $H^{+}$be the half-plane in the direction of the vector $\boldsymbol{w} . p_{j}$ lies in $H^{+}$resp. $H^{-}$if $c_{j}^{k}>c_{j}^{i}$ resp. $c_{j}^{i}>c_{j}^{k}$.

If $n$ is even then exactly $n / 2$ adjacent half-lines from $\left(p_{j}\right)_{j=1}^{n}$ lie in $H^{+}$and $n / 2$ adjacent half-lines lie in $H^{-}$, see Figure 4 . If $n$ is odd then $\lfloor n / 2\rfloor$ or $\lceil n / 2\rceil$ adjacent half-lines lie in $H^{+}$and the rest of them lie in $H^{-}$, see Figure $4 \mathrm{~b}$.

The next lemma shows that every $3 \mathrm{D}$ visibility representation of a complete graph contains a large short-distance subset. The following sections focus on these subsets.

Lemma 3. Let $\left\{P_{i}=P+\boldsymbol{w}_{\boldsymbol{i}}, i=1, \ldots, m\right\}$ be a set of regular $n$-gons. If $\left\{P_{i}\right\}$ is a $3 D$ visibility representation of a complete graph $K_{m}$ then $\left\{P_{i}\right\}$ contains a short-distance subset with at least $\left\lceil\mathrm{m} / 16 n^{2}\right\rceil$ polygons.

Proof. Every two polygons $P_{j}, P_{k}$ from the representation have to intersect (to see each other). Polygons $\left\{P_{i}\right\}$ are shifted copies of $P$ (a polygon inscribed into 
a unit circle). Hence, $P_{j}$ can intersect $P_{k}$ only if the distance of their centers is at most 2. Therefore the set $C$ of centers of polygons from $\left\{P_{i}\right\}$ has the diameter at most 2 .

Let $S$ be a square that contains all points from $C$ and whose side-length is 2 . We can divide this square into $4 n \times 4 n=16 n^{2}$ sub-squares with the sidelength $1 / 2 n$. At least one of these sub-squares must contain at least $\left\lceil m / 16 n^{2}\right\rceil$ points of $C$. We claim that the polygons with the center in this sub-square form a short-distance set.

It is sufficient to show that two points in one sub-square have the distance lower than $\min (\sin (\pi / n), \cos (\pi / n))$. For $x \in(0, \pi / 3\rangle$ we have $\frac{x}{\sqrt{2} \pi}<$ $\min (\sin x, \cos x)$. Hence, for $n \geq 3$ we have $\frac{1}{\sqrt{2} n}<\min (\sin (\pi / n), \cos (\pi / n))$ and $\frac{1}{\sqrt{2} n}$ is the maximum distance of two points in one sub-square.

\section{Regular $2 k$-gons}

The goal of this section is a polynomial upper bound on the maximum size of a complete graph with a $3 \mathrm{D}$ visibility representation by regular $2 k$-gons. We start with a lemma that points out an important forbidden configuration of three polygons.

Lemma 4. Let $\left\{P_{1}, P_{2}, P_{3}\right\}$ be a short-distance set of regular $2 k$-gons. If $\left\{P_{1}\right.$, $\left.P_{2}, P_{3}\right\}$ is a $3 D$ visibility representation of a complete graph $K_{3}$ then it cannot happen that $c_{1}^{1}<c_{1}^{2}<c_{1}^{3}$ and $c_{2}^{1}>c_{2}^{2}>c_{2}^{3}$ (where $\left(c_{j}^{i}\right)_{j=1}^{n}$ are coordinates of $\left.P_{i}\right)$.

Proof. If $c_{1}^{1}<c_{1}^{2}<c_{1}^{3}$ and $c_{2}^{1}>c_{2}^{2}>c_{2}^{3}$ then $c_{l}^{1}>c_{l}^{2}>c_{l}^{3}$ for $l \in\{2, \ldots, k+1\}$ and $c_{l}^{1}<c_{l}^{2}<c_{l}^{3}$ for $l \in\{k+2, \ldots, 2 k\} \cup\{1\}$ by Lemma 2 Therefore, $c_{l}^{2}>$ $\min \left(c_{l}^{1}, c_{l}^{3}\right)$ for $l \in\{1, \ldots, 2 k\}$ and $P_{1}$ cannot see $P_{3}$ according to Lemma 1 but this is a contradiction.

The following lemma shows that if the sequence $\left(c_{1}^{i}\right)_{i}$ of the first coordinates is monotone then the size of the representation is small.

Lemma 5. Let $\left\{P_{i}, i=1, \ldots, m\right\}$ be a short-distance set of regular $2 k$-gons. If $\left\{P_{i}\right\}$ is a $3 D$ visibility representation of a complete graph $K_{m}$ and $\left(c_{1}^{i}\right)_{i=1}^{m}$ is a monotone sequence (where $\left(c_{j}^{i}\right)_{j=1}^{n}$ are coordinates of $P_{i}$ ) then $m \leq k+1$.

Proof. We assume that the sequence $\left(c_{1}^{i}\right)_{i=1}^{m}$ is increasing. The proof for a decreasing sequence is similar. Let $I=\left\{\{i, j\}: i<j, c_{2}^{i}>c_{2}^{j}\right\}$, i.e., the pairs of polygons whose boundaries intersect in $\overline{m_{1} c m_{2}}$. We claim that $I=\emptyset$ or $\bigcap I \neq \emptyset$.

We proceed by contradiction. Let's assume that $I \neq \emptyset$ and $\bigcap I=\emptyset$. At first we show that there must be (at least) two disjoint pairs in $I$. Let's assume that there aren't two disjoint pairs in $I$. If $\{a, \bar{a}: a<\bar{a}\} \in I$ then there exist $B=\{b, \bar{b}: b<\bar{b}\}$ and $C=\{c, \bar{c}: c<\bar{c}\}$ in $I$ such that $a \notin B$ and $\bar{a} \notin C$ (because $a, \bar{a} \notin \bigcap I$ ). Moreover $\bar{a} \in B$ and $a \in C$ because the pairs $\{a, \bar{a}\}$ and $B$ (resp. $C$ ) are not disjoint. If $\bar{a}=b$ then $c_{1}^{a}<c_{1}^{\bar{a}}=c_{1}^{b}<c_{1}^{\bar{b}}$ and $c_{2}^{a}>c_{2}^{\bar{a}}=c_{2}^{b}>c_{2}^{\bar{b}}$ which is in contradiction with Lemma 4. Therefore $\bar{a}=\bar{b}$ and $B=\{b, \bar{a}\}$. 
An analogous argument shows that $a=c$ and $C=\{a, \bar{c}\}$. The pairs $B$ and $C$ are not disjoint according to our assumption. This can happen only if $\bar{c}=b$ but then $c_{1}^{a}<c_{1}^{\bar{c}}=c_{1}^{b}<c_{1}^{\bar{a}}$ and $c_{2}^{a}>c_{2}^{\bar{c}}=c_{2}^{b}>c_{2}^{\bar{a}}$ which is in contradiction with Lemma 4 again. This means that there must be two disjoint pairs in $I$.

Let $\{a, \bar{a}: a<\bar{a}\}$ and $\{b, \bar{b}: b<\bar{b}\}$ be disjoint pairs in $I$. We can assume without loss of generality that $a<b$.

Let's assume that $\bar{a}<\bar{b}$ (see Figure 5 ):

$$
\begin{gathered}
a<\bar{a}<\bar{b}, a<b<\bar{b},\left(c_{1}^{i}\right)_{i} \text { increasing } \Rightarrow c_{1}^{a}<c_{1}^{\bar{a}}<c_{1}^{\bar{b}}, c_{1}^{a}<c_{1}^{b}<c_{1}^{\bar{b}} \\
\{a, \bar{a}: a<\bar{a}\},\{b, \bar{b}: b<\bar{b}\} \in I \Rightarrow c_{2}^{a}>c_{2}^{\bar{a}}, c_{2}^{b}>c_{2}^{\bar{b}} \\
c_{1}^{b}<c_{1}^{\bar{b}}, c_{2}^{b}>c_{2}^{\bar{b}} \Rightarrow c_{l}^{b}>c_{l}^{\bar{b}}, l \in\{2, \ldots, k+1\} \text { by Lemma } 2 \\
c_{1}^{a}<c_{1}^{\bar{a}}, c_{2}^{a}>c_{2}^{\bar{a}} \Rightarrow c_{l}^{a}<c_{l}^{\bar{a}}, l \in\{k+2, \ldots, 2 k\} \cup\{1\} \text { by Lemma } 2 \\
c_{1}^{\bar{a}}<c_{1}^{\bar{b}} \Rightarrow c_{k+1}^{\bar{b}}<c_{k+1}^{\bar{a}} \text { by Lemma } 2
\end{gathered}
$$

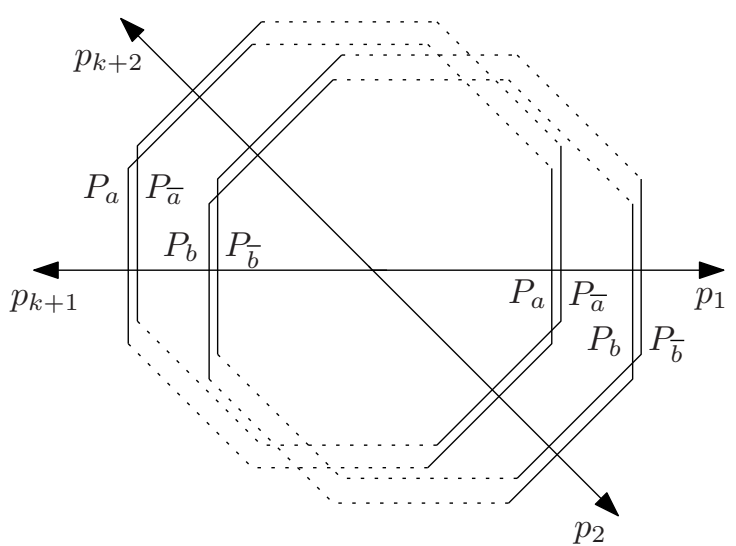

Fig. 5.

We can see that $c_{1}^{a}<c_{1}^{b}$ and $c_{l}^{b}>c_{l}^{\bar{b}}, l \in\{2, \ldots, k+1\}$. Therefore $c_{l}^{b}>\min \left(c_{l}^{a}, c_{l}^{\bar{b}}\right)$, $l \in\{1, \ldots, k+1\}$. Similarly, $c_{k+1}^{\bar{b}}<c_{k+1}^{\bar{a}}$ and $c_{l}^{a}<c_{l}^{\bar{a}}, l \in\{k+2, \ldots, 2 k\} \cup$ $\{1\}$, i.e., $c_{l}^{\bar{a}}>\min \left(c_{l}^{a}, c_{l}^{\bar{b}}\right), l \in\{k+1, \ldots, 2 k\} \cup\{1\}$. Hence, $P_{a}$ cannot see $P_{\bar{b}}$ according to Lemma 1 but this cannot happen because $\left\{P_{i}\right\}$ is a representation of a complete graph. Therefore, it cannot be $\bar{a}<\bar{b}$.

If $\bar{b}<\bar{a}$ then $a<b<\bar{b}<\bar{a}$ and $c_{1}^{a}<c_{1}^{b}<c_{1}^{\bar{b}}<c_{1}^{\bar{a}}$ because $\left(c_{1}^{i}\right)_{i}$ is increasing. $c_{2}^{\bar{a}}<c_{2}^{a}$ and $c_{2}^{\bar{b}}<c_{2}^{b}$ because $\{a, \bar{a}: a<\bar{a}\},\{b, \bar{b}: b<\bar{b}\} \in I$. If $c_{2}^{\bar{a}}<c_{2}^{\bar{b}}$ then $P_{b}, P_{\bar{b}}$ and $P_{\bar{a}}$ are in contradiction with Lemma 4 . Similarly, if $c_{2}^{b}<c_{2}^{a}$ then $P_{a}, P_{b}$ and $P_{\bar{b}}$ are in contradiction with Lemma 4 . Therefore it must be $c_{2}^{\bar{b}}<c_{2}^{\bar{a}}<c_{2}^{a}<c_{2}^{b}$ but this means that the disjoint pairs $\{a, \bar{b}: a<\bar{b}\}$, $\{b, \bar{a}: b<\bar{a}\}$ satisfy assumptions of the previous paragraph and we again have a contradiction with the completeness of the represented graph. 
We know that $\bar{a} \neq \bar{b}$ because $\{a, \bar{a}\}$ and $\{b, \bar{b}\}$ are disjoint. On the other hand, both possibilities $\bar{a}<\bar{b}$ and $\bar{b}<\bar{a}$ lead to a contradiction. Hence, the original assumption that $I \neq \emptyset$ and $\bigcap I=\emptyset$ cannot be satisfied. It must be either $I=\emptyset$ or $\bigcap \neq \neq \emptyset$.

If $I=\emptyset$ then $\left(c_{2}^{i}\right)_{i}$ is increasing. If $I \neq \emptyset$ then there exists $a \in \bigcap I$. This means that if $i<j$ and $c_{i}^{2}>c_{j}^{2}$ then $i=a$ or $j=a$. In other words, the sequence $\left(c_{2}^{i}\right)_{i \in\{1, \ldots, m\} \backslash\{a\}}$ is increasing.

We can repeat this proof with $c_{2}, c_{3}, \ldots, c_{k}$ subsequently and show that there is a set $A$ such that $|A| \leq k$ and $\left(c_{k+1}^{i}\right)_{i \in\{1, \ldots, m\} \backslash A}$ is increasing. On the other hand, this sequence is also decreasing by Lemma 2 because $\left(c_{1}^{i}\right)_{i \in\{1, \ldots, m\} \backslash A}$ is increasing. Therefore the sequence $\left(c_{k+1}^{i}\right)_{i \in\{1, \ldots, m\} \backslash A}$ has length at most 1 and $1 \geq|\{1, \ldots, m\} \backslash A| \geq m-k$.

Now we are ready to prove the main theorem of this section.

Theorem 1. If $\left\{P_{i}, i=1, \ldots, m\right\}$ is a $3 D$ visibility representation of a complete graph $K_{m}$ by regular $n$-gons (where $n=2 k$ ) then $m \leq 4 n^{2}(n+2)^{2}$.

Proof. The set $\left\{P_{i}\right\}$ contains a short-distance subset $\left\{P_{i}^{\prime}\right\}$ with at least $\left\lceil m / 16 n^{2}\right\rceil$ polygons according to Lemma 3 , Let $\left(c_{j}^{i}\right)_{j=1}^{n}$ be coordinates of $P_{i}^{\prime}$. If $\left\lceil m / 16 n^{2}\right\rceil \geq$ $(k+1)^{2}+1$ then due to Erdös-Szekeres theorem [9] the sequence $\left(c_{1}^{i}\right)_{i=1}^{\left\lceil m / 16 n^{2}\right\rceil}$ contains a monotone subsequence of length $k+2$ which is in contradiction with Lemma 5. Therefore $m / 16 n^{2} \leq\left\lceil m / 16 n^{2}\right\rceil \leq(k+1)^{2}$.

\section{Regular $(2 k+1)$-gons}

We focus on regular $(2 k+1)$-gons in this section. We prove a theorem analogous to Theorem 1 Unfortunately, Lemma 4 doesn't hold for $(2 k+1)$-gons. We have to use a more complicated version.

Lemma 6. Let $\left\{P_{1}, P_{2}, P_{3}, P_{4}\right\}$ be a short-distance set of regular $(2 k+1)$-gons. If $\left\{P_{i}\right\}$ is a $3 D$ visibility representation of a complete graph $K_{4}$ then it cannot happen that $c_{1}^{1}<c_{1}^{2}<c_{1}^{3}<c_{1}^{4}$ and $c_{2}^{1}>c_{2}^{2}>c_{2}^{3}>c_{2}^{4}$ (where $\left(c_{j}^{i}\right)_{j=1}^{n}$ are coordinates of $\left.P_{i}\right)$.

Proof. If $c_{1}^{1}<c_{1}^{2}<c_{1}^{3}<c_{1}^{4}$ and $c_{2}^{1}>c_{2}^{2}>c_{2}^{3}>c_{2}^{4}$ then $c_{l}^{1}>c_{l}^{2}>c_{l}^{3}>c_{l}^{4}$ for $l \in\{2, \ldots, k+1\}$ and $c_{l}^{1}<c_{l}^{2}<c_{l}^{3}<c_{l}^{4}$ for $l \in\{k+3, \ldots, 2 k+1\} \cup\{1\}$ by Lemma 2. In other words, $c_{l}^{2}>\min \left(c_{l}^{1}, c_{l}^{3}\right)$ and $c_{l}^{3}>\min \left(c_{l}^{2}, c_{l}^{4}\right)$ for $l \in$ $\{1, \ldots, 2 k+1\} \backslash\{k+2\}$.

$P_{1}$ and $P_{3}$ can see each other. Therefore, $c_{k+2}^{2}<\min \left(c_{k+2}^{1}, c_{k+2}^{3}\right)$ according to Lemma 1. Similarly, $c_{k+2}^{3}<\min \left(c_{k+2}^{2}, c_{k+2}^{4}\right)$ because $P_{2}$ and $P_{4}$ can see each other. But this is a contradiction because the first inequality gives us $c_{k+2}^{2}<c_{k+2}^{3}$ while $c_{k+2}^{3}<c_{k+2}^{2}$ by the second inequality.

We need the following consequence of Lemma 6 several times in the sequel. 
Corollary 1. Let $\left\{P_{1}, P_{2}, P_{3}, P_{4}\right\}$ be a short-distance set of regular $(2 k+1)$ gons. If $\left\{P_{i}\right\}$ is a $3 D$ visibility representation of a complete graph $K_{4}$ then it cannot happen that $c_{1}^{1}<c_{1}^{2}<c_{1}^{3}<c_{1}^{4}$ and $c_{k+1}^{1}<c_{k+1}^{2}<c_{k+1}^{3}<c_{k+1}^{4}$ (or $\left.c_{k+2}^{1}<c_{k+2}^{2}<c_{k+2}^{3}<c_{k+2}^{4}\right)$.

Proof. If $c_{1}^{1}<c_{1}^{2}<c_{1}^{3}<c_{1}^{4}$ and $c_{k+1}^{1}<c_{k+1}^{2}<c_{k+1}^{3}<c_{k+1}^{4}$ then $c_{k+2}^{1}>$ $c_{k+2}^{2}>c_{k+2}^{3}>c_{k+2}^{4}$ by Lemma 1 but this is in contradiction with Lemma 6 for coordinates $k+1$ and $k+2$ (Lemma 6 holds for any pair of adjacent coordinates).

Similarly, if $c_{1}^{1}<c_{1}^{2}<c_{1}^{3}<c_{1}^{4}$ and $c_{k+2}^{1}<c_{k+2}^{2}<c_{k+2}^{3}<c_{k+2}^{4}$ then $c_{k+1}^{1}>$ $c_{k+1}^{2}>c_{k+1}^{3}>c_{k+1}^{4}$ by Lemma 1 and we have a contradiction again.

The next lemma is an analogy of Lemma 5. The proof of this lemma is more complicated because the representations by $(2 k+1)$-gons are more complicated but the main ideas of both proofs (of Lemma [5] and Lemma 7) are the same.

Lemma 7. Let $\left\{P_{i}, i=1, \ldots, m\right\}$ be a short-distance set of regular $(2 k+1)$-gons. There exists $c>0$ independent of $k$ such that if $\left\{P_{i}\right\}$ is a $3 D$ visibility representation of a complete graph $K_{m}$ and $\left(c_{1}^{i}\right)_{i=1}^{m}$ is a monotone sequence (where $\left(c_{j}^{i}\right)_{j=1}^{n}$ are coordinates of $\left.P_{i}\right)$ then $m \leq c k$.

Proof. We assume that the sequence $\left(c_{1}^{i}\right)_{i=1}^{m}$ is increasing. The proof for a decreasing sequence is similar. Let $I=\left\{\{i, j\}: i<j, c_{2}^{i}>c_{2}^{j}\right\}$. We claim that there exists $n_{0} \in \mathbb{N}$ (independent of $k$ ) such that $I$ doesn't contain $n_{0}$ pairwise disjoint pairs.

Let's assume that $J \subseteq I: \forall A, B \in J, A \neq B \Rightarrow A \cap B=\emptyset$. Consider a complete graph on the vertex set $J$. We color the edge $\{\{a, \bar{a}: a<\bar{a}\},\{b, \bar{b}: b<\bar{b}\}: a<b\}$ by

- color 1 when $\bar{a}<\bar{b}$ and $c_{k+2}^{\bar{a}}<\min \left(c_{k+2}^{a}, c_{k+2}^{\bar{b}}\right)$

- color 2 when $\bar{a}<\bar{b}$ and $c_{k+2}^{\bar{a}}>\min \left(c_{k+2}^{a}, c_{k+2}^{\bar{b}}\right)$

- color 3 when $\bar{b}<\bar{a}, c_{2}^{a}<c_{2}^{b}, c_{2}^{\bar{b}}<c_{2}^{\bar{a}}$ and $c_{k+2}^{\bar{b}}<\min \left(c_{k+2}^{a}, c_{k+2}^{\bar{a}}\right)$

- color 4 when $\bar{b}<\bar{a}, c_{2}^{a}<c_{2}^{b}, c_{2}^{\bar{b}}<c_{2}^{\bar{a}}$ and $c_{k+2}^{\bar{b}}>\min \left(c_{k+2}^{a}, c_{k+2}^{\bar{a}}\right)$

- color 5 when $\bar{b}<\bar{a}, c_{2}^{a}<c_{2}^{b}$ and $c_{2}^{\bar{a}}<c_{2}^{\bar{b}}$

- color 6 when $\bar{b}<\bar{a}, c_{2}^{b}<c_{2}^{a}$ and $c_{2}^{\bar{b}}<c_{2}^{\bar{a}}$

- color 7 when $\bar{b}<\bar{a}, c_{2}^{b}<c_{2}^{a}$ and $c_{2}^{\bar{a}}<c_{2}^{\bar{b}}$

If $\{\{a, \bar{a}: a<\bar{a}\},\{b, \bar{b}: b<\bar{b}\}: a<b\}$ has the 7 th color then $c_{1}^{a}<c_{1}^{b}<c_{1}^{\bar{b}}<c_{1}^{\bar{a}}$ because $a<b<\bar{b}<\bar{a}$ and $\left(c_{1}^{i}\right)_{i}$ is increasing. $c_{2}^{\bar{b}}<c_{2}^{b}$ because $\{b, \bar{b}: b<$ $\bar{b}\} \in I$. Therefore $c_{2}^{\bar{a}}<c_{2}^{\bar{b}}<c_{2}^{b}<c_{2}^{a}$ and $P_{a}, P_{b}, P_{\bar{b}}, P_{\bar{a}}$ are in contradiction with Lemma 6. Hence, the 7 th the color is not used and every edge of $K_{J}$ has one of the first six colors.

According to Ramsey's theorem [1011] there exists $n_{0}$ such that if $|J| \geq n_{0}$ then $K_{J}$ contains a monochromatic subgraph $K_{S}, S=\{\{a, \bar{a}: a<\bar{a}\},\{b, \bar{b}: b<$ $\bar{b}\},\{c, \bar{c}: c<\bar{c}\},\{d, \bar{d}: d<\bar{d}\}: a<b<c<d\}$.

If $K_{S}$ has color 1 then $c_{k+2}^{\bar{a}}<c_{k+2}^{\bar{b}}<c_{k+2}^{\bar{c}}<c_{k+2}^{\bar{d}}, \bar{a}<\bar{b}<\bar{c}<\bar{d}$ and $c_{1}^{\bar{a}}<c_{1}^{\bar{b}}<c_{1}^{\bar{c}}<c_{1}^{\bar{d}}$ (because $\left(c_{1}^{i}\right)_{i}$ is increasing). This is in contradiction with Corollary 1 . 
If $K_{S}$ has color 2 then we have:

$$
\begin{gathered}
a<b<\bar{b}, a<\bar{a}<\bar{b},\left(c_{1}^{i}\right)_{i} \text { increasing } \Rightarrow c_{1}^{a}<c_{1}^{b}<c_{1}^{\bar{b}}, c_{1}^{a}<c_{1}^{\bar{a}}<c_{1}^{\bar{b}} \\
\{a, \bar{a}: a<\bar{a}\},\{b, \bar{b}: b<\bar{b}\} \in I \Rightarrow c_{2}^{\bar{a}}<c_{2}^{a}, c_{2}^{\bar{b}}<c_{2}^{b} \\
c_{1}^{b}<c_{1}^{\bar{b}}, c_{2}^{\bar{b}}<c_{2}^{b} \Rightarrow c_{l}^{\bar{b}}<c_{l}^{b}, l \in\{2, \ldots, k+1\} \text { by Lemma } 2 \\
c_{1}^{a}<c_{1}^{\bar{a}}, c_{2}^{\bar{a}}<c_{2}^{a} \Rightarrow c_{l}^{a}<c_{l}^{\bar{a}}, l \in\{k+3, \ldots, 2 k+1\} \cup\{1\} \text { by Lemma } 2
\end{gathered}
$$

We can see that $c_{1}^{a}<c_{1}^{b}$ and $c_{l}^{\bar{b}}<c_{l}^{b}, l \in\{2, \ldots, k+1\}$. Hence, $c_{l}^{b}>\min \left(c_{l}^{a}, c_{l}^{\bar{b}}\right)$ for $l \in\{1, \ldots, k+1\}$. Similarly, $c_{l}^{a}<c_{l}^{\bar{a}}, l \in\{k+3, \ldots, 2 k+1\} \cup\{1\}$ and $c_{k+2}^{\bar{a}}>$ $\min \left(c_{k+2}^{a}, c_{k+2}^{\bar{b}}\right)$. Therefore $c_{l}^{\bar{a}}>\min \left(c_{l}^{a}, c_{l}^{\bar{b}}\right)$ for $l \in\{k+2, \ldots, 2 k+1\} \cup\{1\}$. If $c_{k+1}^{\bar{a}}>\min \left(c_{k+1}^{a}, c_{k+1}^{\bar{b}}\right)$ then $P_{a}$ cannot see $P_{\bar{b}}$ according to Lemma 1. It must be $c_{k+1}^{\bar{a}}<\min \left(c_{k+1}^{a}, c_{k \pm 1}^{\bar{b}}\right)$, namely $c_{k+1}^{\bar{a}}<c_{k+1}^{\bar{b}}$. The same argument shows that also $c_{k+1}^{\bar{b}}<c_{k+1}^{\bar{c}}<c_{k+1}^{\bar{d}}$. On the other hand, $c_{1}^{\bar{a}}<c_{1}^{\bar{b}}<c_{1}^{\bar{c}}<c_{1}^{\bar{d}}$ (because $\bar{a}<\bar{b}<\bar{c}<\bar{d}$ ) which is in contradiction with Corollary 1 .

If $K_{S}$ has color 3 then $c_{k+2}^{\bar{d}}<c_{k+2}^{\bar{c}}<c_{k+2}^{\bar{b}}<c_{k+2}^{\bar{a}}, \bar{d}<\bar{c}<\bar{b}<\bar{a}$ and $c_{1}^{\bar{d}}<c_{1}^{\bar{c}}<c_{1}^{\bar{b}}<c_{1}^{\bar{a}}$ (because $\left(c_{1}^{i}\right)_{i}$ is increasing) and we have a contradiction again.

If $K_{S}$ has color 4 then we proceed in a similar way as with the second color. We have:

$$
\begin{gathered}
c_{2}^{a}<c_{2}^{b}, c_{2}^{\bar{b}}<c_{2}^{\bar{a}} \\
a<b<\bar{b}<\bar{a},\left(c_{1}^{i}\right)_{i} \text { increasing } \Rightarrow c_{1}^{a}<c_{1}^{b}<c_{1}^{\bar{b}}<c_{1}^{\bar{a}} \\
\{a, \bar{a}: a<\bar{a}\} \in I \Rightarrow c_{2}^{\bar{a}}<c_{2}^{a} \\
c_{1}^{b}<c_{1}^{\bar{a}}, c_{2}^{\bar{a}}<c_{2}^{a}<c_{2}^{b} \Rightarrow c_{l}^{\bar{a}}<c_{l}^{b}, l \in\{2, \ldots, k+1\} \text { by Lemma } 2 \\
c_{1}^{a}<c_{1}^{\bar{b}}, c_{2}^{\bar{b}}<c_{2}^{\bar{a}}<c_{2}^{a} \Rightarrow c_{l}^{a}<c_{l}^{\bar{b}}, l \in\{k+3, \ldots, 2 k+1\} \cup\{1\} \text { by Lemma } 2
\end{gathered}
$$

We can see that $c_{1}^{a}<c_{1}^{b}$ and $c_{l}^{\bar{a}}<c_{l}^{b}, l \in\{2, \ldots, k+1\}$. Hence, $c_{l}^{b}>\min \left(c_{l}^{a}, c_{l}^{\bar{a}}\right)$ for $l \in\{1, \ldots, k+1\}$. Similarly, $c_{l}^{a}<c_{l}^{\bar{b}}, l \in\{k+3, \ldots, 2 k+1\} \cup\{1\}$ and $c_{k+2}^{\bar{b}}>$ $\min \left(c_{k+2}^{a}, c_{k+2}^{\bar{a}}\right)$. Therefore, $c_{l}^{\bar{b}}>\min \left(c_{l}^{a}, c_{l}^{\bar{a}}\right)$ for $l \in\{k+2, \ldots, 2 k+1\} \cup\{1\}$. If $c_{k+1}^{\bar{b}}>\min \left(c_{k+1}^{a}, c_{k+1}^{\bar{a}}\right)$ then $P_{a}$ cannot see $P_{\bar{a}}$ according to Lemma 1 . It must be $c_{k+1}^{\bar{b}}<\min \left(c_{k+1}^{a}, c_{k+1}^{\bar{a}}\right)$, namely $c_{k+1}^{\bar{b}}<c_{k+1}^{\bar{a}}$. The same argument shows that also $c_{k+1}^{\bar{d}}<c_{k+1}^{\bar{c}}<c_{k+1}^{\bar{b}}$. On the other hand, $c_{1}^{\bar{d}}<c_{1}^{\bar{c}}<c_{1}^{\bar{b}}<c_{1}^{\bar{a}}$ (because $\bar{d}<\bar{c}<\bar{b}<\bar{a}$ ) which is in contradiction with Corollary 1 .

If $K_{S}$ has color 5 then $c_{2}^{\bar{a}}<c_{2}^{\bar{b}}<c_{2}^{\bar{c}}<c_{2}^{\bar{d}}, \bar{d}<\bar{c}<\bar{b}<\bar{a}$ and $c_{1}^{\bar{d}}<c_{1}^{\bar{c}}<c_{1}^{\bar{b}}<c_{1}^{\bar{a}}$ (because $\left(c_{1}^{i}\right)_{i}$ is increasing). This is in contradiction with Lemma 6 .

If $K_{S}$ has color 6 then $c_{2}^{d}<c_{2}^{c}<c_{2}^{b}<c_{2}^{a}$, $a<b<c<d$ and $c_{1}^{a}<c_{1}^{b}<c_{1}^{c}<c_{1}^{d}$ (because $\left(c_{1}^{i}\right)_{i}$ is increasing) and we have a contradiction with Lemma 6 again.

We can see that $K_{J}$ cannot contain a monochromatic subgraph $K_{S}$. Therefore $|J| \leq n_{0}-1$, i.e., $I$ doesn't contain $n_{0}$ pairwise disjoint pairs.

Let $J_{\max } \subseteq I$ be a maximal subset of pairwise disjoint pairs. We know that $\left|\bigcup J_{\max }\right|=2\left|J_{\max }\right| \leq 2\left(n_{0}-1\right)$. For any $A \in I$ there exists $B \in J_{\max }$ such that $A \cap B \neq \emptyset$. Hence, the sequence $\left(c_{2}^{i}\right)_{i \in\{1, \ldots, m\} \backslash \bigcup J_{\max }}$ is increasing. 
We can repeat this proof with $c_{2}, c_{3}, \ldots, c_{k}$ subsequently and show that there is a set $J^{\prime}$ such that $\left|J^{\prime}\right| \leq 2\left(n_{0}-1\right) k$ and $\left(c_{k+1}^{i}\right)_{i \in\{1, \ldots, m\} \backslash J^{\prime}}$ is increasing. The sequence $\left(c_{1}^{i}\right)_{i \in\{1, \ldots, m\} \backslash J^{\prime}}$ is also increasing. Therefore, its length is less than 4 by Corollary [1.e., $4>\left|\{1, \ldots, m\} \backslash J^{\prime}\right| \geq m-2\left(n_{0}-1\right) k$.

Lemma 7 allows us to prove an analogy of Theorem 1 for regular $(2 k+1)$-gons.

Theorem 2. There exists $c>0$ such that if $\left\{P_{i}, i=1, \ldots, m\right\}$ is a $3 D$ visibility representation of a complete graph $K_{m}$ by regular $n$-gons (where $n=2 k+1$ ) then $m \leq c n^{4}$.

Proof. The proof is the same as the proof of Theorem 1 (using Lemma 7 instead of Lemma 5).

If we combine Theorem 1 and Theorem 2 then we obtain the following result.

Theorem 3. If $s(n)$ is the maximum size of a complete graph with a $3 D$ visibility representation by equal regular $n$-gons then $s(n)=O\left(n^{4}\right)$.

Proof. Theorem 1 if $n$ is even and Theorem 2 if $n$ is odd.

\section{Conclusion}

We show that the maximum size of a complete graph with a $3 \mathrm{D}$ visibility representation by regular $n$-gons is $O\left(n^{4}\right)$. This result is a significant improvement of the previously known exponential bound $\left(\begin{array}{c}6 n-3 \\ 3 n-1\end{array}\right)-3 \approx 2^{6 n}$ from [1. We don't attempt to minimize constants in this estimate because there still remains a big gap between the lower bound $\Omega(n)$ and our upper bound $O\left(n^{4}\right)$.

\section{References}

1. Štola, J.: 3D Visibility Representations of Complete Graphs. In: Liotta, G. (ed.) GD 2003. LNCS, vol. 2912, pp. 226-237. Springer, Heidelberg (2004)

2. Babilon, R., Nyklová, H., Pangrác, O., Vondrák, J.: Visibility Representations of Complete Graphs. In: Kratochvíl, J. (ed.) GD 1999. LNCS, vol. 1731, pp. 333-340. Springer, Heidelberg (1999)

3. Fekete, S.P., Houle, M.E., Whitesides, S.: New Results on a Visibility Representation of Graphs in 3D. In: Brandenburg, F.J. (ed.) GD 1995. LNCS, vol. 1027, pp. 234-241. Springer, Heidelberg (1996)

4. Bose, P., Everett, H., Fekete, S.P., Lubiw, A., Meijer, H., Romanik., K., Shermer, T., Whitesides, S.: On a visibility representation for graphs in three dimensions. In: Di Battista, G., Eades, P., de Fraysseix, H., Rosenstiehl, P., Tamassia, R. (eds.) GD 1993, pp. 38-39 (1993)

5. Štola, J.: Unimaximal Sequences of Pairs in Rectangle Visibility Drawing. In: Tollis, I.G., Patrignani, M. (eds.) GD 2008. LNCS, vol. 5417, pp. 61-66. Springer, Heidelberg (2009)

6. Fekete, S.P., Meijer, H.: Rectangle and box visibility graphs in 3D. Int. J. Comput. Geom. Appl. 97, 1-28 (1997) 
7. Tamassia, R., Tollis, I.G.: A unifed approach to visibility representations of planar graphs. Discrete and Computational Geometry 1, 321-341 (1986)

8. Dean, A.M., Hutchinson, J.P.: Rectangle-Visibility Representations of Bipartite Graphs. In: Tamassia, R., Tollis, I.G. (eds.) GD 1994. LNCS, vol. 894, pp. 159166. Springer, Heidelberg (1995)

9. Erdős, P., Szekeres, G.: A combinatorial problem in geometry. Compositio Math. 2, 463-470 (1935)

10. Ramsey, F.P.: On a problem of formal logic. Proc. London Math. Soc. 30, 264-286 (1930)

11. Graham, R., Rothschild, B., Spencer, J.: Ramsey Theory, 2nd edn. WileyInterscience Series in Discrete Mathematics. Wiley, New York (1990) 